\title{
Enhanced Size-Dependent Piezoelectricity in Nanostructured $\mathrm{TiO}_{2}$ Films
}

\author{
V. Dallacasa \\ Department of Computer Science, University of Verona, Strada Le Grazie, 15, 37134 Verona, Italy \\ Correspondence should be addressed to V. Dallacasa, valerio.dallacasa@univr.it \\ Received 25 January 2012; Accepted 16 February 2012 \\ Academic Editors: Y. Masuda and R. Mota \\ Copyright () 2012 V. Dallacasa. This is an open access article distributed under the Creative Commons Attribution License, which \\ permits unrestricted use, distribution, and reproduction in any medium, provided the original work is properly cited. \\ We have developed a nanogenerator that is driven by mechanical forces to produce continuous direct-current output. The \\ nanogenerator was fabricated with titanium dioxide nanoparticle arrays forming a Schottky barrier with a conducting electrode \\ with a small gap. Under uniaxial mechanical compression, nanogenerators have shown repeatable and consistent electrical outputs \\ with energy-conversion efficiency of order of magnitude at least comparable to similar nanogenerators based on piezoelectric \\ materials. Flexoelectricity due to inhomogeneous strain induced in the nanostructured $\mathrm{TiO}_{2}$ film has been identified as one \\ possible mechanism of the high apparent piezoelectricity in the nanoparticles. The approach presents an adaptable, mobile, and \\ cost-effective technology for harvesting mechanical energy from the environment. At the present stage it offers a potential solution \\ for powering nanodevices.
}

\section{Introduction}

Recently, an approach for converting mechanical energy into electrical energy has been suggested by using piezoelectric zinc oxide $(\mathrm{ZnO})$ nanowire arrays $[1,2]$. This technique has illustrated the feasibility of utilizing inorganic nanomaterials with semiconducting and piezoelectric properties for nanogenerators. The conversion of energy can be achieved by creating a Schottky junction between $\mathrm{ZnO}$ and a metallic corrugated zigzag electrode which realizes a rectifying contact. Results have been obtained with ultrasound waves with typical output powers $\mathrm{P} \sim 1 \mathrm{pW} / \mathrm{mm}^{2}$ and sound waves with typical intensity 100 decibels which generate electricity in the order of $1-50 \mathrm{mV}$. It is believed that various novel applications including devices for the recharging of mobile phones, the operation of micro and nanodevices, and for the energy storage in batteries and capacitors become possible if prototype devices can be optimized to enhance their power input. It has been estimated that the performance can be improved by two-three orders of magnitude [2].

In this paper we report results of $\mathrm{TiO}_{2}$-based nanogenerators operated under static mechanical forces. The output power of such nanogenerators can be attributed to piezoelectricity in the $\mathrm{TiO}_{2}$ nanoparticles due to the flexoelectric effect which is expected at the nanoscale [3-6] for systems which are non-piezoelectric in bulk form.

\section{Generator Design and Fabrication}

2.1. Design. The overall layout of the generator is shown in Figure 1. A sandwich structure is obtained with an array of aligned $\mathrm{TiO}_{2}$ nanostructures of films between two conducting ITO/glass electrodes. The ITO coating enhanced the conductivity of the electrodes and at the same time created a Schottky contact at the interface of $\mathrm{TiO}_{2}$ and the top electrode.

2.2. Fabrication Process. The device was fabricated by the deposition of a nanostructured $\mathrm{TiO}_{2}$ film on a ITO/glass substrate that forms an electrode. A colloidal paste was prepared by starting from nanostructured commercial powders (Degussa) [7]. To optimize the separation of $\mathrm{TiO}_{2}$ clusters, mechanical machining in aqueous phase, in organic phase, or both and/or sonication of a concentrated emulsion of $\mathrm{TiO}_{2}$ was used. Such emulsion was then applied to the ITO/glass substrates (Optical Filters Ltd., England). The deposition was achieved by a dr. blade or spray deposition 


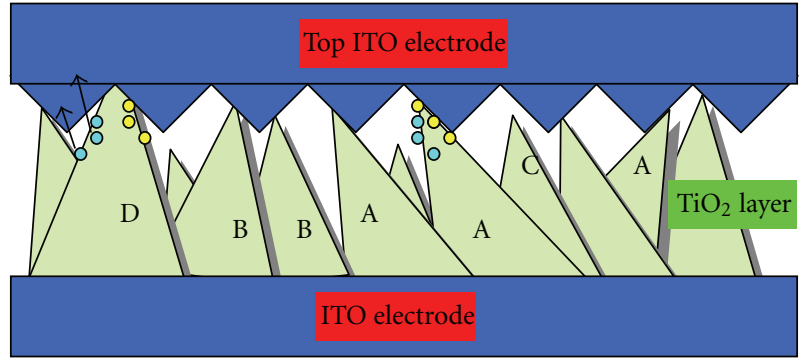

○ Negative charge

○ Positive charge

Figure 1: Setup of the generator. Nanoparticles are schematically described by cusps that can be bended/compressed by the top electrode.

method. To optimize the homogeneity of the film structure and its conductivity, a sintering procedure was used by heating the devices overnight at a temperature $T=550^{\circ} \mathrm{C}$. With this technique, films with typical thickness $10-20 \mu \mathrm{m}$ are obtained.

Detailed optical microscope inspection indicate that the final $\mathrm{TiO}_{2}$ films present a uniform distribution with tightly sinterized grains.

AFM microscopy indicates that at the $\mu \mathrm{m}$ scale the films are constituted by a disordered array in the $x y$ plane with cusps (Figure 2). At larger resolution they exhibit a nanometric structure with nanocrystals having comparable dimension $\sim 30 \mathrm{~nm}$ within the $x y$ plane and in the $z$-direction, if they were distributed compactly, a surface density $10^{12} \mathrm{~cm}^{-2}$ on the average would result.

A similar nanostructure is revealed for the ITO electrode, with slightly smaller density $10^{10} \mathrm{~cm}^{-2}$ and diameter and height $100 \mathrm{~nm}$ and $5 \mathrm{~nm}$, respectively (Figure 3 ).

Each of the structures appears as aligned nanocrystals distributed irregularly in size and orientation strongly resembling the configuration shown in Figure 1.

The top electrode was an ITO/glass identical to the substrate and completed the sandwiched structure.

Typical dimensions of the devices are or order $5 \times 5 \mathrm{~cm}^{2}$.

The commercial ITO/glass substrates and top electrodes (Optical Filters Ltd., England) have typical surface resistance $20 \Omega$ and a resistivity $\rho=20 \cdot 10^{-4} \Omega \mathrm{cm}$, such values being compatible with those reported in the literature [8].

When used as the substrate for deposition of the oxide, the ITO structure acts as a template to the arrangement of the oxide on it. Good adhesion is obtained for $\mathrm{TiO}_{2}$ films indicating a good crystal structure matching.

From the other hand, when ITO is used as the top electrode, its nanometric array structure is penetrated on contact by the smaller $\mathrm{TiO}_{2}$ array leading to an optimized contact and good mechanical interaction with the film.

\section{The Operating Principle}

The physical principle for creating charges on the nanoparticles is a coupling of piezoelectric and semiconducting

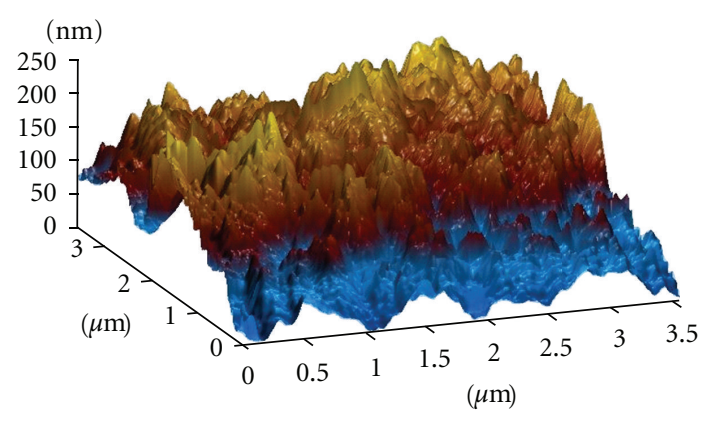

Figure 2: AFM scan of $\mathrm{TiO}_{2}$ films at the micron scale. Nanoparticles are schematically described by cusps.

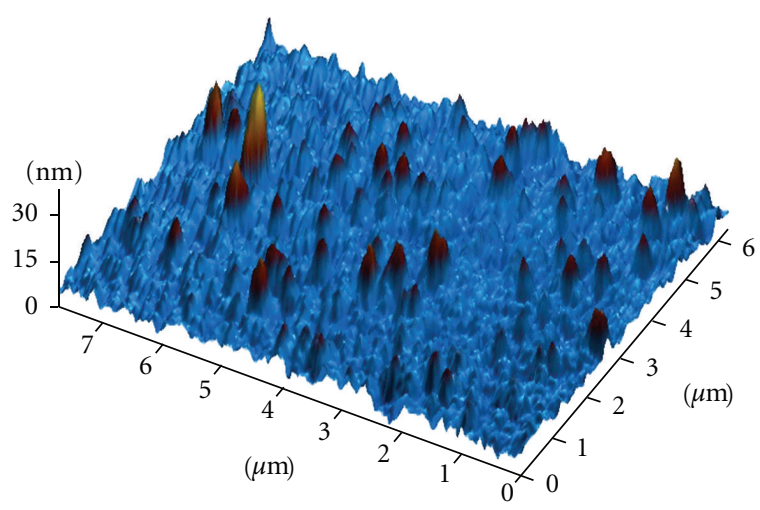

Figure 3: AFM scan of ITO conducting films on glass. A nanometric structure is apparent.

properties [1]. Under external stress by the top electrode, a strain field is created in the nanoparticles. This results in an electric field along the $z$-direction inside the nanoparticle and on its surface through the piezoelectric effect. Under compression/dilation, the electric potential distribution is approximately between $\mathrm{V}-(\mathrm{V}+)$ and $\mathrm{V}+(\mathrm{V}-)$ towards the top electrode.

The potential is created by the relative displacement of the Ti cations with respect to the $\mathrm{O}_{2}$ anions in the crystal structure; these ionic charges cannot freely move and cannot recombine without releasing the strain. The potential difference is maintained as long as the deformation is in place and no foreign free charges (such as from the ITO contact) are injected.

The positive (negative) voltage created by the charges acts as an applied bias on the top electrode leading to polarization of the Schottky diode at the interface and passage of current through the barrier. The current is the result of $\Delta V$ driven flow of electrons between the electrode and the semiconductor $\mathrm{TiO}_{2}$. The flow of the free electrons through the nanoparticle will neutralize the ionic charges distributed in the volume and on the surface of the nanoparticles and thus reduce the magnitudes of the potentials $\mathrm{V}+$ and $\mathrm{V}-$. 


\section{Measuring System}

When the top electrode has a configuration as shown in Figure 1, by scanning the electrode back and forth, we can reproduce the processes discussed above. Under uniaxial stress, the electrode will exert both vertical and lateral forces on the nanoparticles, and there will be generation of electricity. During the scan nanoparticles may either be bent on making contact with the electrode (A- and D-type in Figure 1) or contact the electrode without being bent (Btype) or remain isolated without any contact (C-type). The $\mathrm{B}$ nanoparticles constitute a parallel resistance of the device; the $\mathrm{C}$ nanoparticles are an infinite resistance not entering in the process.

The ITO electrode in contact with the $\mathrm{TiO}_{2}$ array was manipulated by a probe station. Pressure was applied on the sandwich through a calibrated force gauge (Lutron Electronics) of capacity $49 \mathrm{~N}$. The voltage and resistance have been measured by an high-impedance voltmeter (Kitley).

A typical device configuration was studied by positioning the ITO electrode on the nanostructure, and applying uniaxial compression stress the spacing was controlled to obtain a reasonable contact by the monitoring of the resistance of the junction. The pressing head of the gauge was of cylindrical form of diameter $d=5 \mathrm{~mm}$. In order to distribute the force over the whole junction, a rigid layer is interposed between the head and the top electrode. The actual area of the junction which is compressed is estimated not larger than $1 \mathrm{~cm}^{2}$, slightly larger than the head dimension due to the lateral propagation of the applied force but smaller than the area of the whole device. Variations of pressure in time can be obtained by manipulating the head of the gauge manually or electronically to study the response of the devices both in static and dynamical conditions. In the following we report results under uniaxial static compression.

\section{Results}

In Figure 4, we report the measured voltage at the junction as a function of the applied force for $\Delta F<6 \mathrm{~N}$. The voltage exhibits a linear dependence at lower force, an indication of charge accumulation at the interface at increased pressing levels. At larger forces it departs from such linear law and finally degrades to the $\mathrm{mV}$ range, which can indicate enhanced charge screening.

A decrease of the junction resistance is also observed, indicating an enhanced carrier mobility trough the junction. A clear correlation of the trends of voltage and resistance with the sign of the stress results. Furthermore, the voltage at the top electrode has always positive (negative) sign with increasing (decreasing) force, a typical evidence of a piezoelectric effect. Spontaneous voltages $\Delta V \sim 0.5 \mathrm{~V}$ are measured at nominally zero stress, which can be ascribed to residual compressing stress internal to the nanograins of the device. An example of measured values of the resistance and its fitting curve is reported in Figure 5.

Large fluctuations (from sample to sample and in the same sample) appear in the voltage and the resistance, accompanied by time relaxation towards equilibrium as well as

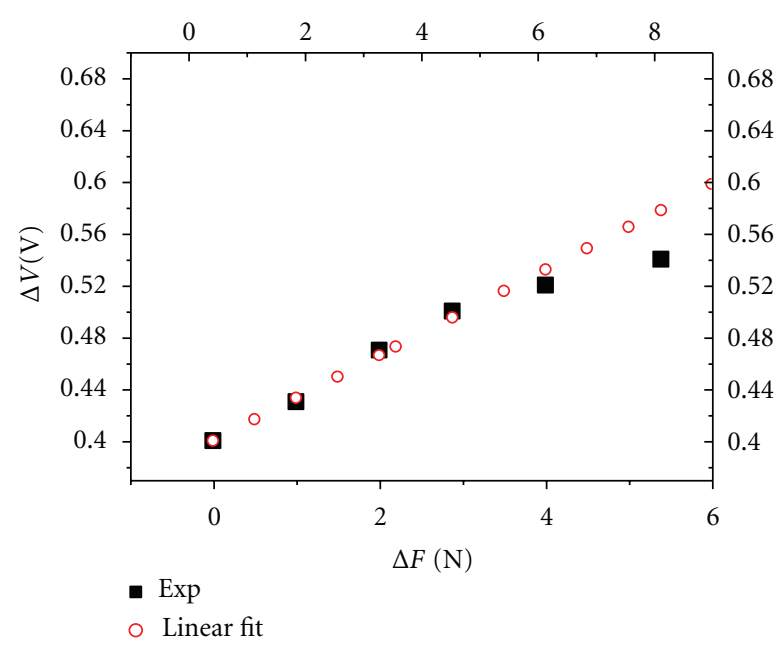

FIGURE 4: Induced voltage change versus applied force (static).

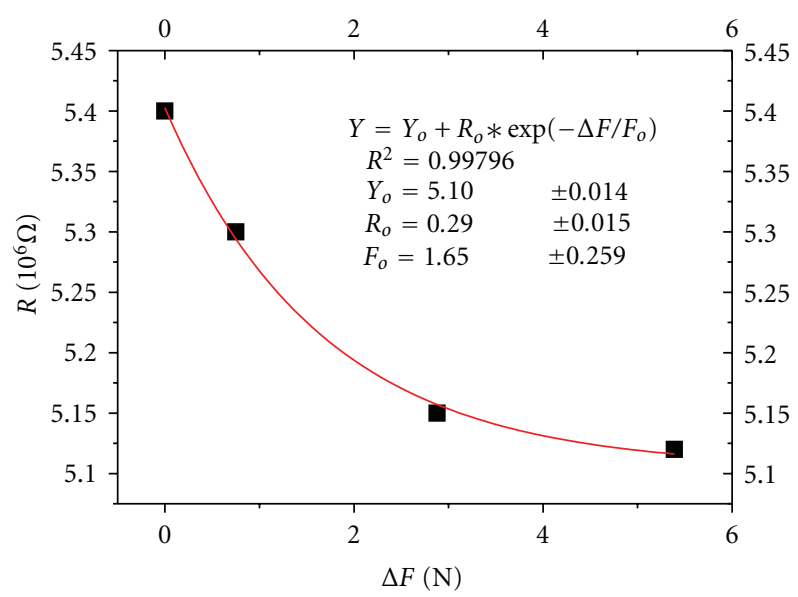

FIGURE 5: Induced resistance change versus applied force (static).

large hysteretic behaviour. Typically, times of order of several minutes can be required to achieve stabilized values. These fluctuations arise from the setup of the junctions and are determined by the pressing condition of the top electrode on the oxide film.

The principal results obtained with such devices and the geometrical and electric parameters are summarized in Table 1.

The lower contact on the ITO/glass substrate may be assumed to be ohmic, as indicated by the employed deposition method, which is a standard one in the literature [7].

The capacitance of the system is ignored in the circuit in order to simplify the discussion about DC measurement. The capacitance of the junctions turns out to be of order of magnitude $C \sim 10^{-10} \mathrm{~F}$.

\section{Discussion}

We can explain our results by piezoelectricity in nanoparticles produced by the flexoelectric effect [3-6]. 
TABLE 1: Geometric parameters of device and film nanoparticles.

\begin{tabular}{lcc}
\hline & $\mathrm{TiO}_{2}$ (this work) & $\mathrm{ZnO}[2]$ \\
\hline Area $\left(\mathrm{cm}^{2}\right)$ & 10 & $2 \cdot 10^{-2}$ \\
Density $\left(\mathrm{cm}^{-2}\right)$ & $10^{11}$ & $10^{9}$ \\
Height $(\mathrm{nm})$ & 10 & $10^{3}$ \\
Diameter $(\mathrm{nm})$ & 10 & 40 \\
Film thickness $(\mu \mathrm{m})$ & $20-30$ & \\
Resistance $(\Omega)$ & $5 \cdot 10^{6}$ & $3.5 \cdot 10^{3}$ \\
Voltage $(\mathrm{V})$ & $0.5($ static$)$ & $\sim 0$ \\
Power $(\mathrm{W})$ & $10^{-3}($ dynamic $)$ & $\sim 10^{-3}$ \\
\hline
\end{tabular}

Centrosymmetric dielectrics are not expected to polarize under mechanical strain. A nonuniform strain or the presence of strain gradients can, however, locally break inversion symmetry and induce polarization even in centrosymmetric crystals. This phenomenon is termed flexoelectricity [3-6].

With reference to Figure 1, at moderate pressure nanoparticles are deformed and acquire positive and negative charges on the surface along the compression direction. The positive charges in contact with the top electrode turn out to produce an applied forward bias at the Schottky barrier so that current will flow at the junction. On traversing the junction from ITO, electrons will screen the piezo charges at the interface thereby decreasing the potential. The effect will be higher the higher the polarization, that is the current at the junction.

In order to examine the existence of the barrier, we have used reported values of the work function and electron affinity of ITO and $\mathrm{TiO}_{2}$ respectively. Reported data for the work function of ITO are in the range $4.17-5.54 \mathrm{eV}$ with an average $4.6-4.7 \mathrm{eV}[9,10]$. Reported data for the electron affinity of $\mathrm{TiO}_{2}$ in nanoporous films are in the range $3.9-4.3 \mathrm{eV}$ [11-13]. We thus expect a barrier of the order $0.5-0.6 \mathrm{eV}$, which compares with the results of the voltages measured at the junction.

The order of magnitude of the piezoelectric constant can be obtained from the accumulated charge by modeling the junction as a condenser. The piezoelectric constant $d$ can be defined by the equation $\Delta Q=d \Delta F$, where $\Delta Q$ and $\Delta F$ are the piezoelectric induced charge and the applied force respectively. The electric potential can be written as $\Delta V=$ $Q L / A \varepsilon_{o} \varepsilon_{r}$, where $A$ is the area of the junction, $L$ the film thickness, and $Q$ the total piezoelectric charge so that

$$
\Delta V=d \Delta F \frac{L}{A \varepsilon_{o} \varepsilon_{r}} .
$$

The quantity $\Delta V / \Delta F$ can be deduced from the slope of experimental curve in Figure 4 which amounts to $\Delta V / \Delta F=$ $3.310^{-2} \mathrm{~V} / \mathrm{N}$. So, for typical values $A \sim 1 \mathrm{~cm}^{2}, \varepsilon_{r}=10, L=$ $10 \mu \mathrm{m}$, we obtain $d=2.9 \times 10^{-11} \mathrm{C} / \mathrm{N}$.

We likewise can derive the value of $d$ from the experimental results on resistance. On modelling the junction by a diode equation, the resistance will be given by

$$
R=R_{\mathrm{o}} \exp \left(\frac{-\Delta V}{V T}\right)
$$

Table 2: Piezoelectric parameters of the $\mathrm{TiO}_{2}$ device.

\begin{tabular}{lc}
\hline$d$ coefficient $(\mathrm{C} / \mathrm{N})$ & $\sim 10^{-11}$ \\
\hline Charge (electron/particle) & $\sim 0.1$ \\
Voltage $(\mathrm{mV})$ & $1-500$ \\
Capacity $(\mathrm{F})$ & $\sim 10^{-10}$ \\
\hline
\end{tabular}

where $V_{T}=m(\mathrm{kT} / \mathrm{e}), m$ being an ideality factor of the diode and $\mathrm{T}$ the absolute temperature, is the thermal voltage and $R_{o}=V_{T} / I_{o}$, with Io the characteristic current parameter value of the diode. Since voltage and force are connected through the linear relation (1), we can transform $\Delta V$ into $\triangle F$ in the exponent in (2), so that $R=R_{o} \exp \left(-\Delta F / F_{o}\right)$ with $F_{o}=V_{T} A \varepsilon_{o} \varepsilon_{r} / d L$. By using typical values as in (1), we find that the value $F_{o}$ corresponds to the order of magnitude evidenced by the fitting in Figure 5 provided $V_{T} \sim 0.05 \mathrm{~V}$ requiring an ideality factor $m \sim 2$. This value is consistently found in these devices.

A summary of results on piezoelectric parameters is reported in Table 2.

\section{Conclusions}

A nanogenerator using $\mathrm{TiO}_{2}$ nanoparticles was developed and tested. The output power response that occurred when the device was exposed to static uniaxial stress was measured and compared to that of a nanogenerator using a $\mathrm{ZnO}$ film. The nanogenerator with $\mathrm{TiO}_{2}$ nanoparticles demonstrated a significant improvement over a nanogenerator without nanoparticles which can be attributed to flexoelectricity at nanoscale.

The number of nanoparticles that was active for producing electricity was limited by the multiple contacts between the nanoparticles and the electrode; thus, the output current was rather small in the present design. The nanogenerator can power few nanodevices which operate at $10 \mathrm{nW}$ [14-16] in our current study.

These technical difficulties could be overcome by an optimized design to improve nanogenerator efficiency by patterned growth of high-quality uniform nanoparticle arrays. A $10^{2}-10^{3}$ factor enhancement of the efficiency can be estimated on the basis of the fraction of active nanoparticles.

\section{Acknowledgments}

We thank Prof. D. Tonelli, Prof. M. Berrettoni, and Dr. E. Scavetta of the Department of Physical and Inorganic Chemistry, University of Bologna, for collaboration and advice on the deposition of oxides.

\section{References}

[1] Z. L. Wang and J. Song, "Piezoelectric nanogenerators based on zinc oxide nanowire arrays," Science, vol. 312, no. 5771, pp. 243-246, 2006.

[2] X. Wang, J. Song, J. Liu, and Z. L. Wang, "Direct-current nanogenerator driven by ultrasonic waves," Science, vol. 316, no. 5821, pp. 102-105, 2007. 
[3] M. S. Majdoub, P. Sharma, and T. Çağin, "Enhanced sizedependent piezoelectricity andelasticity in nanostructure due to the flexoelectric effect "' Physical Review B, vol. 77, no. 12, Article ID 125424, 9 pages, 2008.

[4] M. S. Majdoub, P. Sharma, and T. Çă̆in, "Erratum: enhanced size-dependent piezoelectricity and elasticity in nanostructures due to the flexoelectric effect," Physical Review B, vol. 79, no. 11, Article ID 119904, 2 pages, 2009.

[5] M. S. Majdoub, P. Sharma, and T. Çağin, "Dramatic enhancement in energyharvesting for a narrow range of dimensions in piezoelectric nanostructures," Physical Review B, vol. 78, no. 12, Article ID 121407, 4 pages, 2008.

[6] M. S. Majdoub, P. Sharma, and T. Çağin, "Erratum: dramatic enhancement in energy harvesting for a narrow range of dimensions in piezoelectric nanostructures," Physical Review $B$, vol. 79, no. 15, Article ID 159901, 2 pages, 2009.

[7] V. Dallacasa, F. Dallacasa, P. Di Sia, E. Scavetta, and D. Tonelli, "Nanogenerators based on $\mathrm{ZnO}$ or $\mathrm{TiO}_{2}$ oxides," Journal of Nanoscience and Nanotechnology, vol. 10, no. 2, pp. 1043-1050, 2010.

[8] J. Bregman, Y. Shapira, and H. Aharoni, "Effects of oxygen partial pressure during deposition on the properties of ion-beam-sputtered indium-tin oxide thin films," Journal of Applied Physics, vol. 67, no. 8, pp. 3750-3753, 1990.

[9] Y. Park, V. Choong, Y. Gao, B. R. Hsieh, and C. W. Tang, "Work function of indium tin oxide transparent conductor measured by photoelectron spectroscopy," Applied Physics Letters, vol. 68, no. 19, pp. 2699-2701, 1996.

[10] R. Schlaf, H. Murata, and Z. H. Kafaki, "Work funcion measurement on indium tin oxide films," Journal of Electron Spectroscopy and Related Phenomena, vol. 120, pp. 149-154, 2011.

[11] X. Wang, G. I. Koleilat, J. Tang et al., "Tandem colloidal quantum dot solar cells employing a graded recombination layer," Nature Photonics, vol. 5, no. 8, pp. 480-484, 2011.

[12] R. Könenkamp and I. Rieck, "Electrical properties of schottky diodes on nano-porous $\mathrm{TiO}_{2}$ films," Materials Science and Engineering B, vol. 69, pp. 519-521, 2000.

[13] R. G. D Sharma and M. S. Roy, "Hybrid nano-cristalline $\mathrm{TiO}_{2}$ solar cell with copper phtalocyanine sensitizer and hole transporter," Indian Journal of Pure \& Applied Physics, vol. 49, pp. 557-563, 2011.

[14] Y. Huang, X. Duan, Y. Cui, L. J. Lauhon, K. H. Kim, and C. M. Lieber, "Logic gates and computation from assembled nanowire building blocks," Science, vol. 294, no. 5545, pp. 1313-1317, 2001.

[15] A. Bachtold, P. Hadley, T. Nakanishi, and C. Dekker, "Logic circuits with carbon nanotube transistors," Science, vol. 294, no. 5545, pp. 1317-1320, 2001.

[16] J. Chen, V. Perebeinos, M. Freitag et al., "Bright infrared emission from electrically induced excitons in carbon nanotubes," Science, vol. 310, no. 5751, pp. 1171-1174, 2005. 

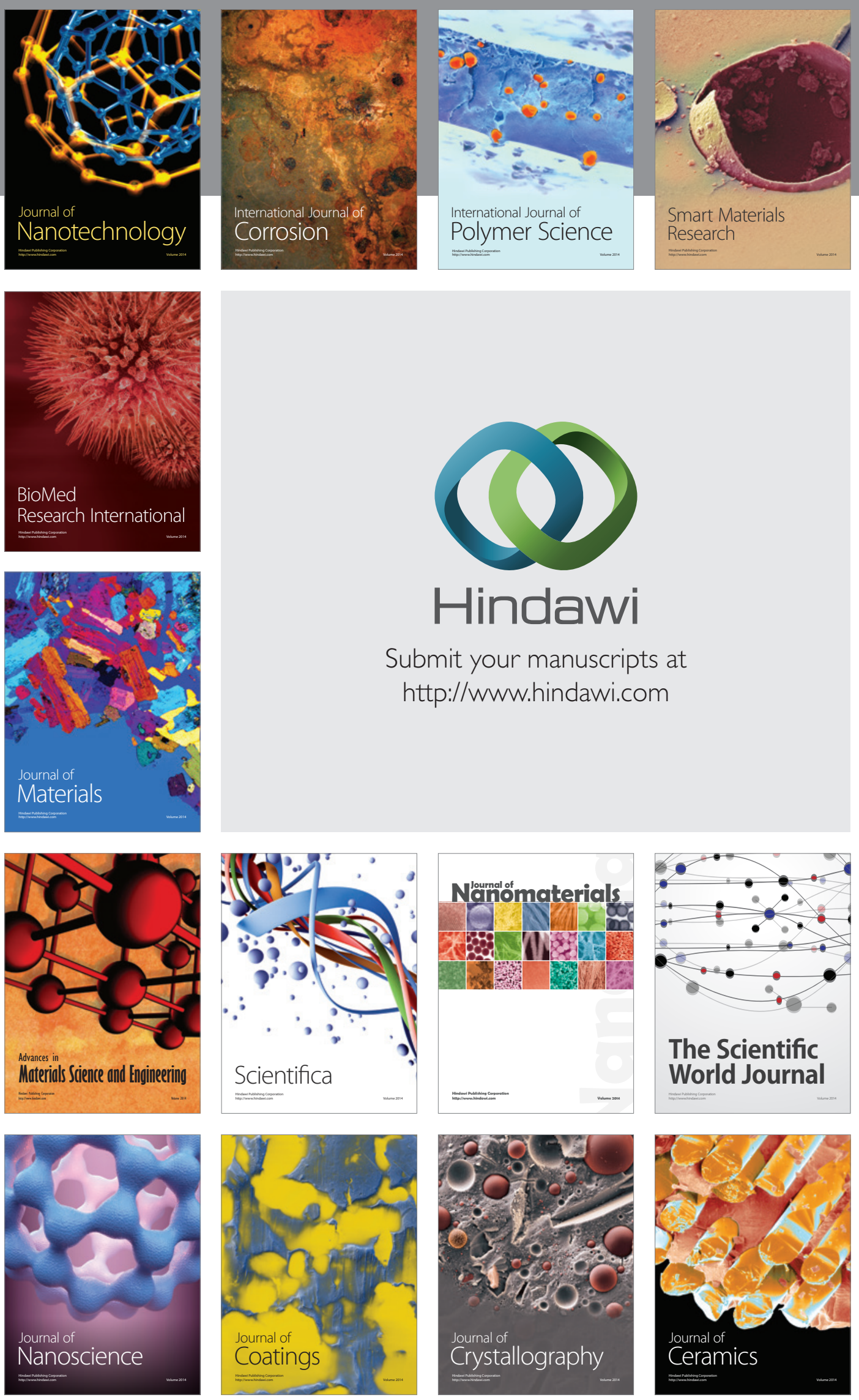

The Scientific World Journal

Submit your manuscripts at

http://www.hindawi.com

\section{World Journal}

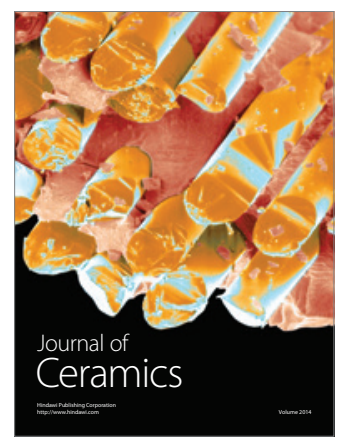

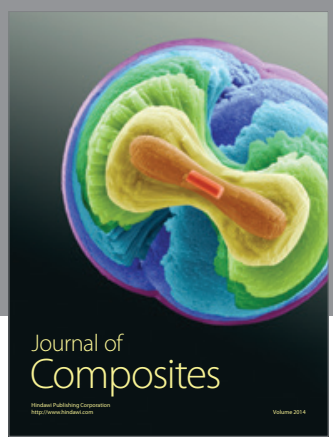
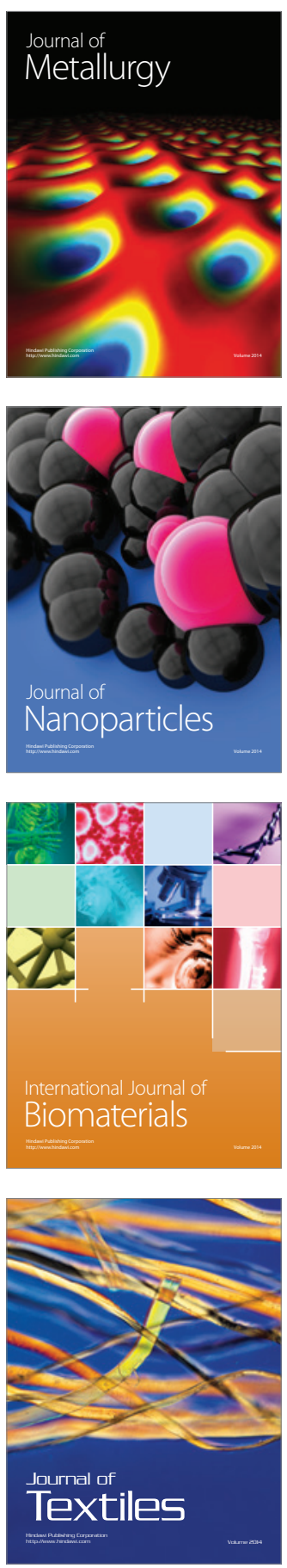